\title{
mTOR inhibitors show promising in vitro activity in bladder cancer and head and neck squamous cell carcinoma
}

\author{
FIONA SCHEDEL ${ }^{1 *}$, RALPH PRIES $^{2 *}$, BEATE THODE $^{1}$, BRIGITTE WOLLMANN $^{2}$, SANDRA WULFF $^{1}$, \\ DIETER JOCHAM ${ }^{1}$, BARBARA WOLLENBERG ${ }^{2}$ and INGO KAUSCH ${ }^{1,3}$
}

\begin{abstract}
Departments of ${ }^{1}$ Urology, and ${ }^{2}$ Otorhinolaryngology, University at Lübeck, University Hospital Schleswig-Holstein, Campus Lübeck, Ratzeburger Allee 160, 23538 Lübeck, Germany
\end{abstract}

Received October 20,2010; Accepted November 22, 2010

DOI: $10.3892 /$ or.2011.1146

\begin{abstract}
Bladder cancer and head and neck squamous cell carcinoma (HNSCC) are frequent but lack efficient therapies especially in advanced disease. Almost no studies on mTOR function and inhibition in these tumor entities have been reported. We examined the gene and protein expression levels of mTOR and its activated form (pmTOR) in three human bladder carcinoma cell lines (RT-4, T24, EJ28) and three HNSCC cell lines (PCI-1, PCI-13, BHY). Furthermore, the consequences of mTOR inhibition by mTOR-specific siRNAs and the MTOR inhibitor temsirolimus were analysed in vitro using immunohistochemical Ki-67 staining, mTOR and pmTOR Western blot analysis, MTT assay, as well as cell cycle analysis with flow cytometry. Especially pmTOR protein expression levels showed marked differences between cell lines. siRNA transfection was associated with dose-dependent target protein reduction but not proliferation inhibition or apoptosis. On the contrary, temsirolimus significantly reduced cell viability and induced apoptosis and cell cycle arrest. According to these data, bladder cancer and HNSCC are promising tumor entities for mTOR inhibition with temsirolimus.
\end{abstract}

\section{Introduction}

Bladder cancers and head and neck squamous cell cancers are frequent tumors. Bladder cancers are the second most

Correspondence to: Dr Ralph Pries, Department of Otorhinolaryngology, University at Lübeck, University Hospital SchleswigHolstein, Campus Lübeck, Ratzeburger Allee 160, 23538 Lübeck, Germany

E-mail: rallepries@yahoo.de

Present address: ${ }^{3}$ Department of Urology and Pediatric Urology, Ammerland Clinic, Westerstede, Germany

${ }^{*}$ Contributed equally

Key words: mTOR, temsirolimus, bladder cancer, head and neck squamous cell carcinoma common urologic malignancy in the Western world with estimated 70,980 new cases diagnosed each year in the Unites States (1) and 85,880 in Europe (2). The gold standard for muscle invasive (>T1) urothelial cancer of the bladder is radical cystectomy with radiochemotherapy as an alternative. While 5-year overall survival rates for localized muscleinvasive bladder cancer after cystectomy are approximately $80 \%, 5$-year survival rates are already significantly poorer after cystectomy in T3a tumors (20-43\%) and especially in lymph node positive patients (17-35\%) (3). Patients with metastatic disease have a mean survival of 3-6 months. The currently best option in these patients is cisplatin-based chemotherapy which results in $10-50 \%$ complete remissions. However, the survival benefit is less than 4 months.

Head and neck squamous cell carcinoma (HNSCC) is the sixth most common tumor occurring almost exclusively among middle-aged tobacco and alcohol abusers (4). Tumors of the oral cavity and pharynx were estimated with 35,720 new cases diagnosed each year in the Unites States (1) and 74,440 in Europe (2). During the past decades, treatments for HNSCC have changed dramatically due to novel approaches such as combined modality therapy and improvements in surgical and radiotherapeutic techniques, resulting in improved function and quality of life of patients with this disease. Nevertheless, despite innovative surgery, new radiation strategies, and the development of novel chemotherapeutic drugs, the overall 5 -year survival rate remained poor and nearly unchanged. Recurrent and/or metastatic HNSCC carries a dismal prognosis with a median survival of about 6 months. The overall response rate with single agent cytotoxics ranges between 15 and $30 \%$. Higher response rates are reported with combination regiments, but better survival has not yet been demonstrated (5).

Especially cells of head and neck cancer are known to develop molecular strategies to escape efficient antitumor immune responses. It is supposed that tumor production of various immunosuppressive mediators contributes to suppressed immune functions. The molecular mechanisms responsible for these malignant transformation processes and the development of the immunosuppressive HNSCC microenvironment remain mostly unknown (5-11).

Due to these poor therapeutic results, preclinical evaluation of torisel alone or in combination with other agents in these tumors seems promising. 
Interestingly, except for initial NIH cell line testing, only one report on preclinical or clinical evaluation of mTOR inhibition has been published for bladder cancer and only three studies have been published including preclinical data on temsirolimus in squamous cell carcinoma of the head and neck. However, the success of current treatment regiments in these tumors is highly limited in cases with locally advanced or metastatic disease. Our aim was to evaluate mTOR inhibition with temsirolimus and specific siRNAs in these tumor entities.

\section{Materials and methods}

Cell culture. Three human bladder carcinoma cell lines (RT4, T24 and EJ28) and three human HNSCC-cell lines (PCI-1, PCI-13 and BHY) were used. The cell lines RT4 and T24 were grown in RPMI-1640 supplemented with 10\% FCS, $1 \%$ sodium pyruvate and penicillin-streptomycin. EJ28, PCI-1, PCI-13 and BHY were cultured in DMEM containing $10 \%$ FCS, 1\% MEM (non-essential amino acids), 1\% HEPES and penicillin-streptomycin. All cells were incubated at $37^{\circ} \mathrm{C}$ in a humidified atmosphere containing $5 \% \mathrm{CO}_{2}$. The mTORinhibitor temsirolimus (CCI-779) (Torise $\left.{ }^{\circledR}\right)$, kindly provided by Wyeth (Wyeth Pharma, Münster, Germany), was stored at $4^{\circ} \mathrm{C}$ and suspended in DMSO the day of use.

Immunohistochemistry/fluorescence microscopy. Immunohistochemical detection of mTOR, phospho-mTOR and Ki-67 was performed on cytospin preparations using the following antibodies: anti-mTOR pS2448 (rabbit) by Rockland (Hamburg, Germany), anti-mTOR (rabbit) by Millipore (MA, USA) anti-human Ki-67 antigen (mouse, clone: MIB-1) by Dako (Hamburg, Germany) and DAPI (4',6-Diamidine2'-phenylindole dihydrochloride) by Roche (Mannheim, Germany). Secondary antibodies were a FITC-labelled goat anti-Rabbit IgG (ZyMax ${ }^{\text {TM }}$ Grade) by Zymed (San Francisco, CA, USA) and a Cy3-labelled goat anti-rabbit antibody (Dianova GmbH, Hamburg, Germany). Replacement of primary antibody with PBS was used as the control.

The photographs were taken with AxioCam MRm Digital Camera (Zeiss, Göttingen, Germany) of the fluorescence microscope Zeiss Axiovert 200M and edited by AxioVision 4.7. For evaluation of antibody-positive fractions the software Image J 1.42q was used; on average a number of 266 cells per slide were counted.

Western blot analysis. Cells were washed with phosphatebuffered saline (PBS), scraped using Laemmli-buffer and lysed by QIAshredder (Qiagen, Hilden, Germany). The protein contents of the samples were determined by Pierce BCA Protein Assay Kit according to the manufacturer's protocol. Cell lysates were separated by electrophoresis through a 7.5\% SDS-PAGE gel for the detection of mTOR and phosphorylated mTOR (pmTOR) respectively a $12.5 \%$ SDSPAGE for $B$-actin control and transferred to a nitrocellulose membrane. The membranes were incubated with mTOR Anti-mTOR (rabbit) (by Millipore, USA) and pmTOR pS2448 antibodies (by Rockland, USA) 1:2500, subsequently incubated with secondary anti-rabbit IgG-coupled HRP antibody 1:50000 (Sigma, A0545, Munich, Germany). Blots were visualized by adding Pierce ECL Western Blotting substrate.

$R T-P C R$. Transfection was performed with appropriate mTOR siRNA (concentration: 1, 10, $100 \mathrm{nM}$ ). Cells were harvested and total RNA was prepared by use of the Quiashredder/ Rneasy Mini Kit (Qiagen). DNA was digested using Dnase I according to the Gibco/Invitrogen protocol (18). For cDNA synthesis $500 \mathrm{ng}$ of total RNA was reverse-transcribed according to the Gibco/Invitrogen protocol real-time PCR was performed with the iCycler (Bio-Rad) PCR Mastermix protocol (qPCR Core Kit for SYBR-Green I, Eurogentec, Belgium).

The 5'-3' primer sequences are: GAPDH: forward, 5'-GAC AGT CAG CCG CAT CTT CTT-3', and reverse, 5'-TCC GTT GAC TCC GAC CTT C-3' (Metabion, Martinsried, Germany); mTOR: forward, 5'-TGC CAA CTA TCT TCG GAA CC-3', reverse, 5'-GCT CGC TTC ACC TCA AAT TC-3' (Metabion).

After an initial activation for $2 \mathrm{~min}$ at $95^{\circ} \mathrm{C}$ the 40 amplification reaction cycles consisted of $95^{\circ} \mathrm{C}$ for $45 \mathrm{sec}, 60^{\circ} \mathrm{C}$ for $45 \mathrm{sec}$. Quantification of gene expression was performed by measuring $\Delta \Delta \mathrm{Ct}$-method. All values represent the mean of duplicates for each concentration.

MTT assay. Cells were seeded in 96-well plates at density of $2 \times 10^{4} /$ well. After $24 \mathrm{~h}$, cells were treated with CCI-779, cisplatin or mTOR siRNA at given concentrations for 24 or $48 \mathrm{~h}$. At the end of the experiment, $5 \mu 1 \mathrm{MTT}$ (3-(4,5dimethylthiazol-2-yl)-2,5-diphenyltetrazolium bromide) (Sigma-Aldrich, Munich) in solution with $95 \mu 1$ RPMI respectively DMEM was added to each well, followed by incubation at $37^{\circ} \mathrm{C}$ for $4 \mathrm{~h}$. The medium was carefully aspirated and $100 \mu 1$ dimethyl sulfoxide (DMSO) was then added to dissolve blue formazan crystals formed in the live cells. Optical density was measured with a spectrometer at $550 \mathrm{~nm}$.

Flow cytometry. Flow cytometric analysis was performed with a FACSCanto ${ }^{\text {TM }}$ flow cytometer using BD FACS Diva software $^{\text {TM }}$ (Becton-Dickinson, Heidelberg, Germany).

siRNA sequences and transfection. Three different small interfering RNAs (siRNAs) targeting the human mTOR were used. The sequences were as follows: siRNA mTOR1: sense 5'-GGC CUA UGG UCG AGA UUU A dTdT-3', anti-sense 5'-UAA AUC UCG ACC AUA GGC C dTdT-3'; siRNA mTOR2 sense 5'-CCU GCU GGA UGC UGA AUU A dTdT-3' anti-sense 5'-UAA UUC AGC AUC CAG CAG G dTdT-3'. siRNA mTOR3 sense 5'-GGA GUC UAC UCG CUU CUA U dTdT-3' anti-sense 5'-AUA GAA GCG AGU AGA CUC C dTdT-3'.

siRNA sequences were purchased from Eurogentec (Cologne, Germany). As a control for transfection efficiency FITC-labelled non-silencing siRNA (Qiagen) was used and fluorescence measured as described (flow cytometry).

Cells $\left(3.5 \times 10^{5}\right)$ were transfected with $0,1,10$ and $100 \mathrm{nM}$ of mTOR siRNA using Lipofectamine 2000 by Invitrogen according to the manufacturer's protocol. Cells were harvested at 24 and $48 \mathrm{~h}$. 
A

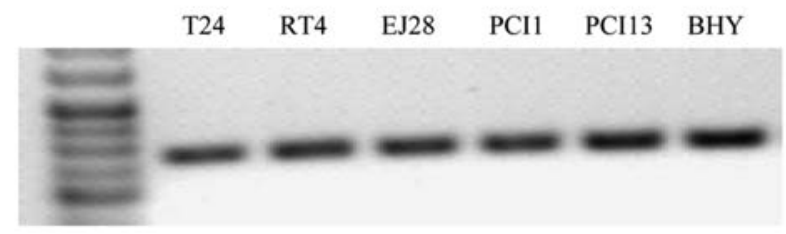

B

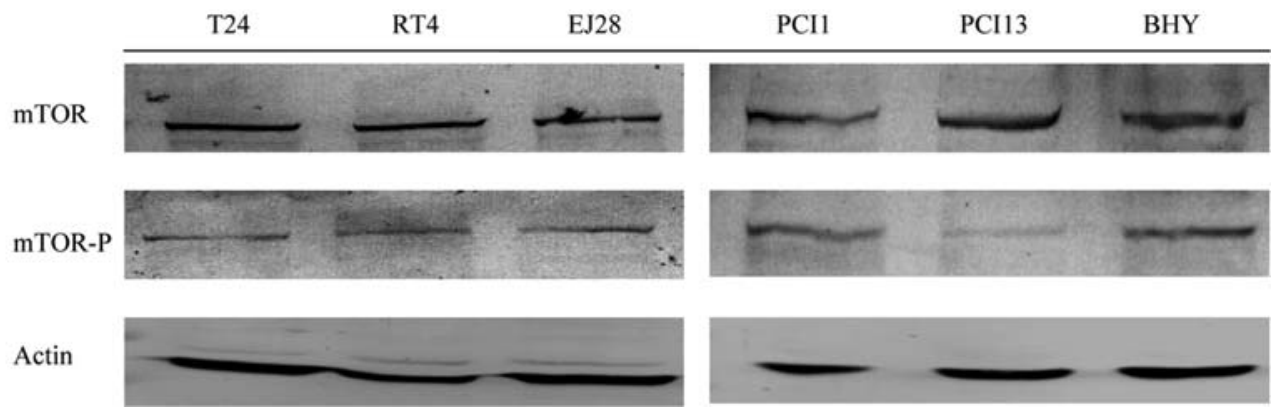

Figure 1. mTOR expression levels in bladder cancer and HNSCC. Expression levels of mTOR were analyzed in different permanent cell lines from bladder cancer (T24, RT4, EJ28) and HNSCC (PCI-1, PCI-13, BHY) on the mRNA (A) and on the protein level (B). (A) Constitutive mTOR mRNA expression was analysed in untreated cells of different cancer cell lines. Gel electrophoresis revealed no marked differences between cell lines. One representative gel is shown. (B) Western blot analysis confirmed the presence of the protein products of both the unphosphorylated as well as phosphorylated form of mTOR in all analyzed cell lines. Actin was used as an internal loading control.

\section{Results}

mTOR expression levels in bladder cancer and HNSCC. We examined the expression of mTOR and its phosphorylated (active) counterpart in various permanent cell lines of bladder cancer (T24, RT4, EJ28) and HNSCC (PCI1, PCI13, BHY). Reverse transcriptase-polymerase chain reaction showed that mTOR is constitutively expressed in all analyzed cell lines with no significant deviations (Fig. 1A). Furthermore, Western blot analysis confirmed the presence of the protein products of both the unphosphorylated as well as phosphorylated form of mTOR in all analyzed cell lines (Fig. 1B). Actin was used as an internal loading control and the cell lines of both tumor entities revealed strong expression levels of phoshorylated mTOR with the exception of HNSCC cell line PCI13 in which lower levels were observed (Fig. 1B). The results suggest that mTOR is highly active in bladder cancer and HNSCC under normal conditions.

siRNA mediates down-regulation of mTOR protein. Three different siRNA constructs against mTOR were used, from which two were newly designed and one was published before (12). Transfection of tumor cells resulted in a dose-dependent protein down-regulation shown in Western blot analysis. Reduced mTOR protein levels in response to siRNA transfection were evaluated in four different cell lines of the two analyzed tumor entities (Bladder cancer: RT-4, T24/HNSCC: PCI-1, PCI-13). Fig. 2 illustrates the protein expression of mTOR and its phosphorylated counterpart.

Best results were achieved using siRNA concentration of $100 \mathrm{nM}$ with the most effective construct range between 39 and $76 \%$ for mTOR, and between 34 and $62 \%$ for pmTOR. Only RT-4 cells were characterized by low inhibition with $<30 \%$. Despite of reduced mTOR translation levels our data

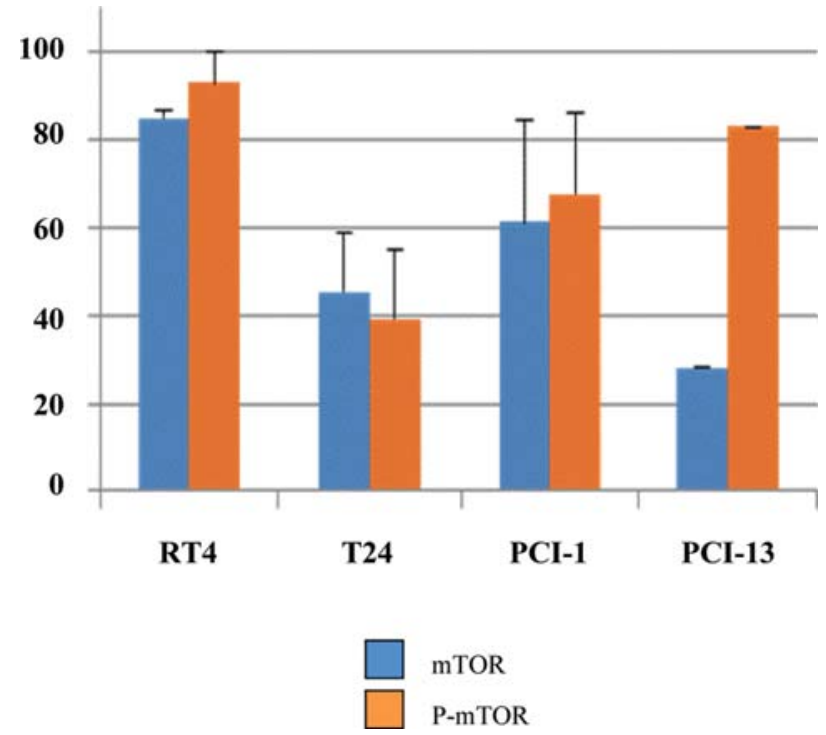

Figure 2. siRNA mediates down-regulation of mTOR and pmTOR protein. Reduced mTOR protein levels in response to siRNA transfection were evaluated in four different cell lines of the two analyzed tumor entities (bladder cancer, RT-4; T24/HNSCC; PCI-1, PCI-13). The relative protein expression levels compared to untreated cells are shown (100\%).

revealed hardly any reduction in levels of active mTOR in the majority of the analyzed cell lines, suggesting a cellular counteraction via increased mTOR phopshorylation (Fig. 2).

Reduced cell proliferation in response to temsirolimus. Cell viability and proliferation of different permanent cell lines of bladder cancer and HNSCC were analyzed in response to mTOR siRNA transfection and temsirolimus treatment, respectively. 
A

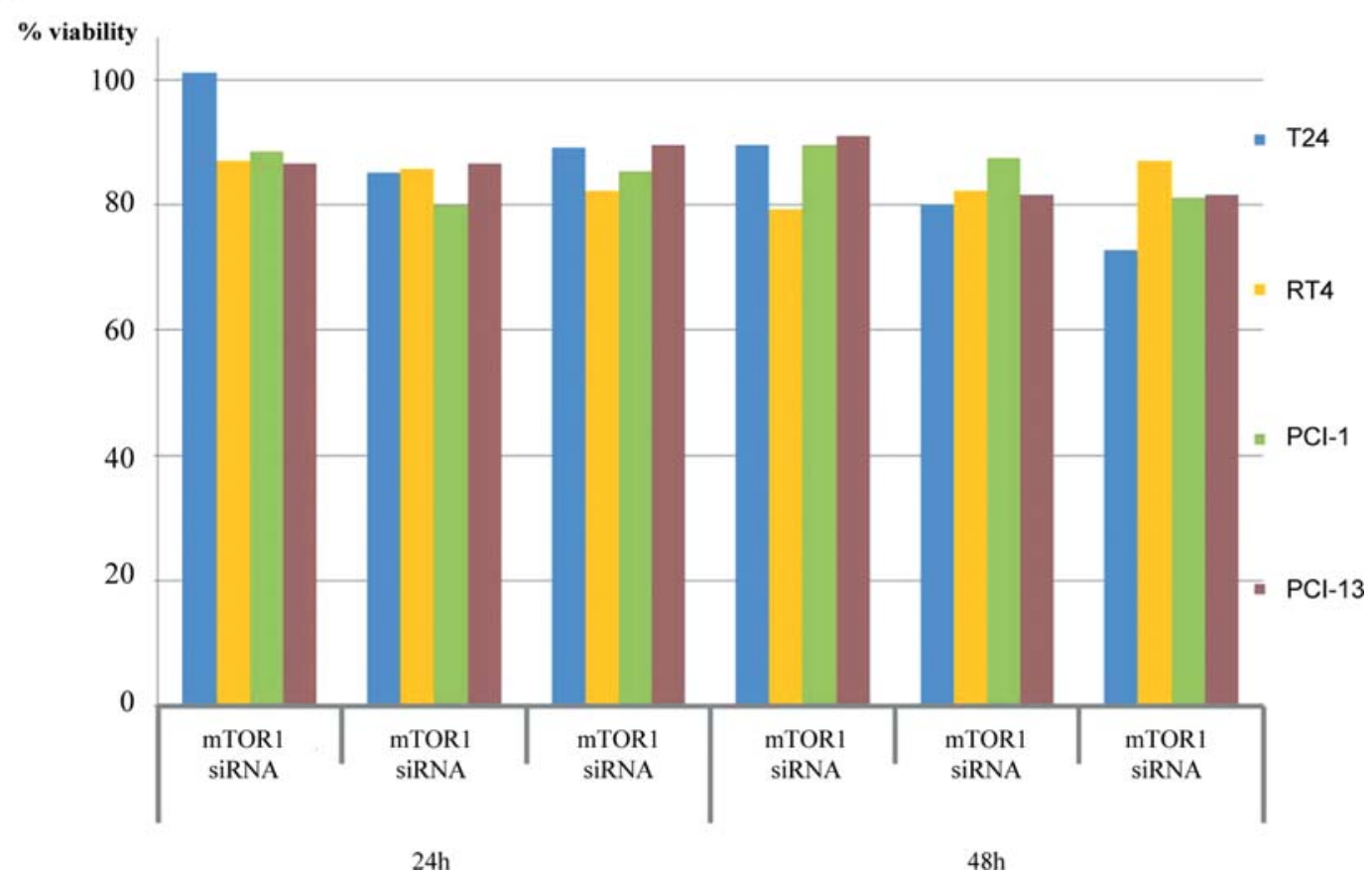

B

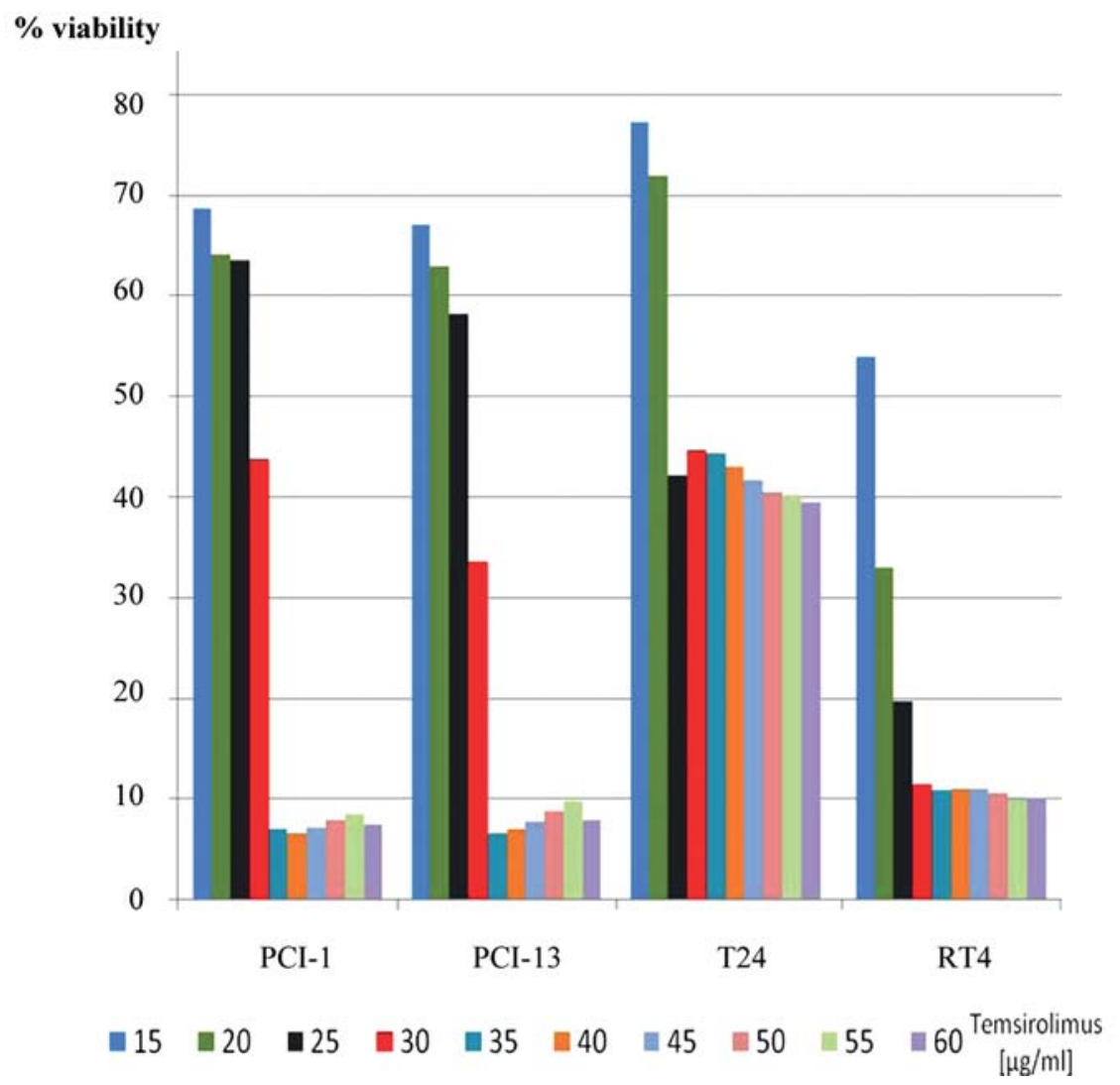

Figure 3. Reduced cell proliferation in response to Temsirolimus. (A) MTT assays revealed no significant influence of the different siRNA constructs on cell growth and viability with any differences between bladder cancer and HNSCC. Four cell lines (T24, RT-4, PCI-1, PCI-13) were examined. (B) In contrast, temsirolimus treatment resulted in strongly reduced cell viability and cell proliferation in all analyzed cell lines. These effects were clearly dose-dependent with a maximum impact between 25 and $60 \mu \mathrm{g}$ temsirolimus per ml. The four cell lines T24, RT-4, PCI-1 and PCI-13 were analysed.

MTT assays revealed no significant influence of the different siRNA constructs on cell growth and viability with any differences between bladder cancer and HNSCC (Fig. 3A). In contrast, temsirolimus treatment resulted in strongly reduced cell viability and cell proliferation in all analyzed cell lines. These effects were clearly dose-dependent with a maximum impact between 25 and $60 \mu \mathrm{g}$ temsirolimus per ml (Fig. 3B). 


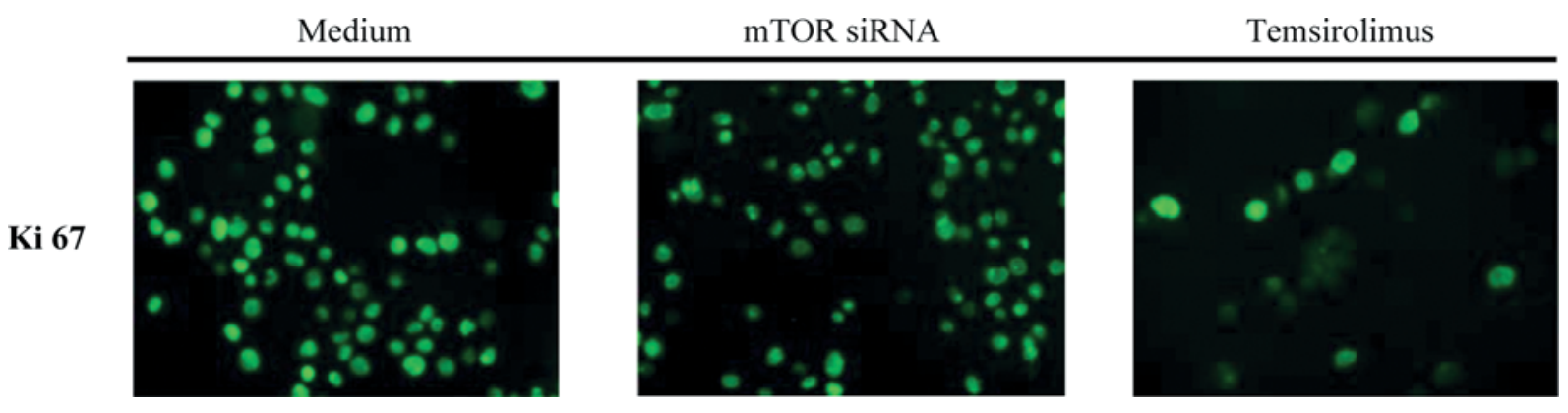

Figure 4. Reduced cell proliferation in response to temsirolimus. In addition, cell proliferation was investigated using immunofluorescence staining of $\mathrm{Ki}-67$. The proliferation status represented by $\mathrm{Ki}-67$ positivity was strongly reduced in response to temsirolimus and not in response to siRNA transfection. PCI-13 cells are shown representatively.

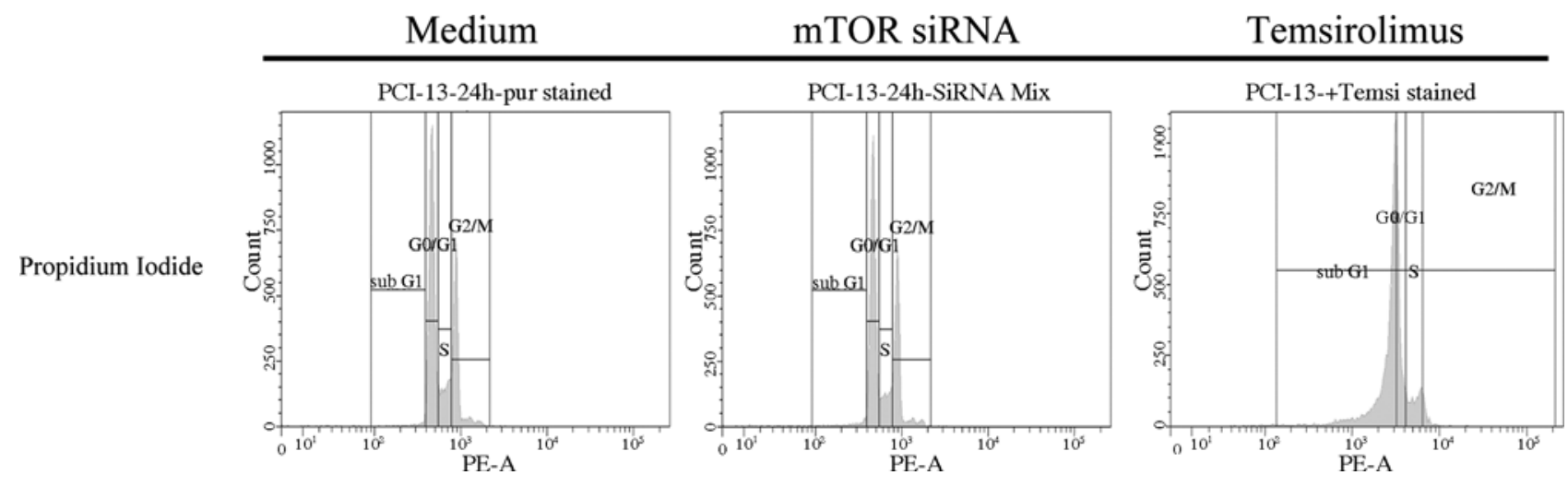

Figure 5. Cell cycle inhibition in response to temsirolimus. Flow cytometric measurements of histograms illustrate that the transfected siRNA constructs have no influence on the cell cycle behavior, whereas temsirolimus clearly leads to apoptosis induction and a G2/M to G0/G1 shift. PCI-13 cells are shown representatively.

In addition, cell proliferation was investigated using immunofluorescence staining of $\mathrm{Ki}-67$. The rate of cell proliferation represented by Ki-67 positivity was strongly reduced in response to temsirolimus and not in response to siRNA transfection (Fig. 4). Temsirolimus reduced cell proliferation on average by $36 \%$ with PCI-13 being the most sensitive cell line (reduction by $51 \%$ ) and T24 the least sensitive (reduction by $20 \%$ ). These data underline the potential of temsirolimus as a promising agent against tumor progression.

Cell cycle inhibition in response to temsirolimus. Flow cytometric measurements with propidium iodide as a DNA selective stain were used to analyze DNA content and cell cycle distribution in response to siRNA transfection and temsirolimus treatment.

The resulting histograms illustrate that the transfected siRNA constructs have no influence on the cell cycle behavior, whereas temsirolimus clearly leads to apoptosis induction and a G2/M to G0/G1 shift (Fig. 5).

\section{Discussion}

Temsirolimus, a novel inhibitor of mammalian target of rapamycin (mTOR), is clinically used in several tumor entities. It has, for instance, demonstrated prolonged overall survival and progression-free survival compared with interferon $\alpha$ (IFN) in patients with advanced renal cell carcinoma (RCC) (13). mTOR is a central element in an evolutionary conserved signalling pathway that regulates proliferation, cell growth, and survival, orchestrating signals originating from growth factors, nutrients or particular stress stimuli. Two important modulators of mTOR activity are the AKT and ERK/MAPK signalling pathways (14). Many studies have shown that mTOR plays an important role in the biology of malignant cells, including deregulation of the cell cycle, inactivation of apoptotic machinery and resistance to chemotherapeutic agents. The development of several mTOR inhibitors, in addition to rapamycin, has facilitated studies of the role of mTOR in cancer, and verified the antitumour effect of mTOR inhibition in many types of neoplasm.

Interestingly, except for initial NIH cell line testing, very few reports on preclinical or clinical evaluation of mTOR inhibition have been published for bladder cancer and for squamous cell carcinoma of the head and neck. However, the success of current treatment regiments in these tumors is highly limited in cases with locally advanced or metastatic disease.

We have found marked mTOR mRNA and mTOR and pmTOR protein levels in several bladder cancer and HNSCC cell lines when compared with RCC cell lines. siRNA inhibition of mTOR was successfully established and the 
effect of mTOR inhibition with temsirolimus and with mTOR siRNAs was examined.

Numerous studies have examined the effect of rapamycin analogues in different tumor entities. An inhibition of proliferation after treatment has repeatedly been described. This could be confirmed in bladder cancer and HNSCC cell lines. Temsirolimus treatment reduced cell viability and cell numbers (data not shown) in MTT tests and in cell growth curves to a much larger extent than mTOR-specific siRNAs. Inhibition of the proliferation fraction demonstrated by Ki-67 staining was found after temsirolimus treatment but not after siRNA treatment.

Furthermore, we have shown in all cell lines a G2/M to G0/G1 shift and an increase of apoptotic cells characterized by the enhanced sub-G1 fraction in histograms.

The first study on inhibition of mTOR as a therapeutic strategy with rapamycin analogues in bladder cancer has recently been published (15). The authors found after treatment of 9 different bladder cancer cell lines with RAD001 (Everolimus) a significant inhibition of proliferation and a cell cycle arrest in the G0/G1 phase without induction of apoptosis. Vega et al have shown that mTOR inhibition with siRNA and rapamycin induced cell cycle arrest in G1 phase with reduction of $\mathrm{S}$-phase fraction and apoptosis in anaplastic lymphoma (16). Peponi et al found cell cycle arrest in G1 after silencing of mTOR with specific siRNAs in mantle cell lymphoma (12). Liu et al examined the level of mTOR expression during cell cycle in poorly differentiated oral squamous carcinoma and in HeLa cells (17). In HNSCC cells mTOR mRNA and protein levels increased in $\mathrm{M}$ phase. In HeLa cells the level of mTOR mRNA and protein did not change during the cell cycle. Activity of mTOR was highest in G2 and M phase. While an early cell cycle arrest after mTOR inhibition has repeatedly been described, data on apoptosis induction is highly heterogeneous. Obviously, effects are very much dependent on tumor entity and potentially on cell differentiation. This will be a central issue of our further investigations.

The results of this study indicate that mTOR inhibition with temsirolimus may be a promising therapeutic approach for bladder cancer and HNSCC.

\section{Acknowledgements}

We are grateful to all members of the Departments of Urology and Otorhinolaryngology for helpful discussions and support. This study was supported by the Wyeth Pharma GmbH.

\section{References}

1. Jemal A, Siegel R, Ward E, Hao Y, Xu J and Thun MJ: Cancer statistics. CA Cancer J Clin 59: 225-249, 2009.

2. ECO/OEC. European Cancer Observatory. International Agency for Cancer. Available from: http://eu-cancer.iarc.fr.; 2009.

3. Kausch I, Doehn C and Jocham D: Recent improvements in the detection and treatment of non-muscle-invasive bladder cancer. Expert Rev Anticancer Ther 6: 1301-1311, 2006.

4. Luce D, Guenel P, Leclerc A, Brugere J, Point D and Rodriguez J: Alcohol and tobacco consumption in cancer of the mouth, pharynx, and larynx: a study of 316 female patients. Laryngoscope 98: 313-316, 1988.

5. Chin D, Boyle GM, Theile DR, Parsons PG and Coman WB: Molecular introduction to head and neck cancer (HNSCC) carcinogenesis. Br J Plast Surg 57: 595-602, 2004.

6. Douglas WG, Tracy E, Tan D, Yu J, Hicks WL Jr, Rigual NR, Loree TR, Wang Y and Baumann H: Development of head and neck squamous cell carcinoma is associated with altered cytokine responsiveness. Mol Cancer Res 2: 585-593, 2004.

7. Pries R, Thiel A, Brocks C and Wollenberg B: Secretion of tumor-promoting and immune suppressive cytokines by cell lines of head and neck squamous cell carcinoma. In Vivo 20: 45-48, 2006.

8. Pries R and Wollenberg B: Cytokines in head and neck cancer. Cytokine Growth Factor Rev 17: 141-146, 2006.

9. Mann EA, Spiro JD, Chen LL and Kreutzer DL: Cytokine expression by head and neck squamous cell carcinomas. Am J Surg 164: 567-573, 1992.

10. Chen Z, Malhotra PS, Thomas GR, Ondrey FG, Duffey DC, Smith CW, Enamorado I, Yeh NT, Kroog GS, Rudy S, McCullagh L, Mousa S, Quezado M, Herscher LL and Van Waes C: Expression of proinflammatory and proangiogenic cytokines in patients with head and neck cancer. Clin Cancer Res 5: 1369-1379, 1999.

11. Woods KV, El-Naggar A, Clayman GL and Grimm EA: Variable expression of cytokines in human head and neck squamous cell carcinoma cell lines and consistent expression in surgical specimens. Cancer Res 58: 3132-3141, 1998.

12. Peponi E, Drakos E, Reyes G, et al: Activation of mammalian target of rapamycin signaling promotes cell cycle progression and protects cells from apoptosis in mantle cell lymphoma. Am J Pathol 169: 2171-2180, 2006.

13. Hudes GR, Berkenblit A, Feingold J, Atkins MB, Rini BI and Dutcher J: Clinical trial experience with temsirolimus in patients with advanced renal cell carcinoma. Semin Oncol 36 (Suppl. 3): 26-36, 2009.

14. Wullschleger $\mathrm{S}$, Loewith $\mathrm{R}$ and Hall MN: TOR signaling in growth and metabolism. Cell 124: 471-484, 2006.

15. Mansure JJ, Nassim R, Chevalier S, Rocha J, Scarlata E and Kassouf W: Inhibition of mammalian target of rapamycin as a therapeutic strategy in the management of bladder cancer. Cancer Biol Ther 8: 2339-2347, 2009.

16. Vega F, Medeiros LF, Vasiliki V, et al: Activation of mammalian target of rapamycin signaling pathway contributes to tumor cell survival in anaplastic lymphoma kinase-positive anaplastic large cell lymphoma. Cancer Res 66: 6589-6597, 2006.

17. Liu Y, Hidayat S, Wen-hui S, Deng X, Yu D and Yu B: Expression and activity of mTOR and its substrates in different cell cycle phases and in oral squamous cell carcinomas of different malignant grade. Cell Biochem Funct 25: 45-53, 2007. 